\title{
Estudio de prevalencia de aneurismas de aorta abdominal y su asociación con factores de riesgo
}

Lederle F , MD, Johnson G, MS et al.Prevalence and associations of abdominal aortic aneurisms detected through screening. Ann Intern Med.1997;126:441-449

\section{Objetivo}

Conocer la prevalencia de aneurismas de aorta abdominal (AAA) e identificar factores de riesgo independientes asociados a esta patología.

\section{Diseño}

Estudio de corte transversal de rastreo.

\section{Lugar}

Quince centros de veteranos de Massachusetts, EEUU

\section{Pacientes}

Se incluyeron 73451 pacientes entre 50 y 79 años sin antecedentes de AAA.

\section{Evaluación de factores de riesgo}

Determinación de factores de riesgo mediante un cuestionario previo al examen ecográfico.

\section{Medición de resultados principales}

Medición de la aorta abdominal con ecografía.

\section{Resultados}

Se detectaron 3366 (4.6\%) AAA mayores de $3 \mathrm{~cm}$ de los cuales 1031 (1.4\%) fueron mayores de $4 \mathrm{~cm}$. y 368 $(0.5 \%)$ mayores de $5 \mathrm{~cm}$. El tabaquismo fue el factor más asociado, para $\mathrm{AAA}>$ de $4 \mathrm{~cm}$. el odds ratio $(\mathrm{OR})$ fue de 5.57 (IC 95\% 4.24 a 7.31 ).

La historia familiar de AAA se encontró asociada, OR 1.95 (IC 95\% 1.56 a 2.43 ).

No se detectaron AAA > de $4 \mathrm{~cm}$. en menores de 60 años. Después de los 75 años, la prevalencia fue cuatro veces mayor que en la década de los sesenta años. Las enfermedades arterioescleróticas (cardiovascular, cerebrovascular y enfermedad vascular periférica) se encontraron asociadas OR 1.68 (IC 95\% 1.47 a 1.92) para AAA > de $4 \mathrm{~cm}$. La hipertensión mostró una muy débil relación OR 1.16 ( IC 95\% 1.01 a 1.32).

Se encontró relación inversa con diabetes, sexo femenino y un examen ecográfico previo en los últimos 5 años.

\section{Conclusiones}

La prevalencia encontrada en este estudio es probablemente representativa de la población general. El tabaquismo fue el factor más fuertemente asociado con AAA, y probablemente exista una relación causal. La edad, presencia de enfermedad coronaria u otra arterioesclerótica, dislipemia e historia familiar de AAA mostraron una importante asociación; mientras que la diabetes y el sexo femenino tienen una relación negativa. Coherente con resultados de estudios previos, la hipertensión presentó una asociación marginal. Los datos obtenidos de este estudio proveen información de gran importancia para planificar futuros programas de rastreo de AAA; pero queda todavía por resolver si estos programas son costo efectivos y si logran reducir la morbi-mortalidad.

\section{Comentario}

Los AAA son una patología de alto impacto; ya que existe una importante diferencia de mortalidad entre la ruptura aneurismática y la cirugía electiva previa al evento. El presente estudio es el más grande, pero no refleja completamente a la población general. Los veteranos de guerra de EEUU son casi exclusivamente hombres, la historia de tabaquismo es superior a la media y se trata en general de una población más enferma.

El hallazgo de AAA mayores de 5 $\mathrm{cm}$ (los que tienen indicación quirúrgica) en 1 de cada 200 pacientes da muestra de la magnitud del hallazgo. El estudio excluye los pacientes con antecedentes de AAA, por lo cual la prevalencia es probablemente mayor; lamentablemente estos datos no son reportados. El hallazgo de tabaquismo como primer factor asociado, debería comparar- se con la población general en que la historia de tabaquismo es significativamente menor. La relación con la historia familiar, si bien es dificil de evaluar en un cuestionario, probablemente sea mayor ya que recién hace pocos años que esta patología se reconoce como entidad patológica definida. Estudios recientes muestran que la probabilidad de encontrar AAA es 18 veces mayor en miembros de la familia que en la población general (2). La asociación con cardiopatía isquémica debe hacernos considerar otro aspecto para decidir el rastreo que es la mortalidad del tratamiento quirúrgico; otros estudios muestran que un $18 \%$ de pacientes con AAA previo a cirugia electiva poseen enfermedad coronaria severa asintomática y el $31 \%$ de las muertes en el postoperatorio in mediato son por infarto de miocardio (1). El hallazgo de AAA al reali- zar una ecografia previa por cualquier otra causa es interesante. Se calcula que podría hacerse detección de casos de esta forma a un $12-15 \%$ de las personas susceptibles, disminuyendo así la utilización de recur$\operatorname{sos}(3)$.

Dr. Augusto Granel Unidad de Medicina Familiar y Preventiva Hospital Italiano de Buenos Aires

\section{Bibliografía:}

1.Ernst $\mathrm{Cb}$. Abdominal aortic aneurysm.N Engl.J Med 1993;328:1167-1172

2.Limet.R.Familial risk for abdominal aortic aneurysm and its consequences for organization of selective detection.J.Mal.Vasc 1995;20(4):258-7

3.Derbyshire et al. Opportunistic screening for abdominal aortic aneurysms. 4.Med.Screeen.1994;1(4):220-2 